\title{
Preoperative Idoxuridine and Radiation For Large Soft Tissue Sarcomas: Clinical Results With Five-Year Follow-Up
}

\author{
Vernon K. Sondak, MD, John M. Robertson, MD, Jeffrey J. Sussman, MD, Patricia A. Saran, RN, \\ Alfred E. Chang, MD, and Theodore S. Lawrence, MD
}

\begin{abstract}
Background: Local control remains an important issue in the management of large soft tissue sarcomas. Radiation is the main adjuvant to surgery for local therapy of sarcomas, but it requires relatively high doses, hitherto considered prohibitive in areas such as the retroperitoneum. We developed a preoperative treatment approach to large soft tissue sarcomas that would deliver a high total dose of radiation administered in conjunction with the halogenated pyrimidine radiosensitizer idoxuridine (IdUrd).

Methods: Thirty-seven patients with large sarcomas of the head and neck, mediastinum, retroperitoneum, or extremity received three or five cycles of sequential IdUrd infusion (1000-1600 $\mathrm{mg} / \mathrm{m}^{2} / \mathrm{d} \times 5 \mathrm{~d}$ ) alternating weekly with twice daily radiation $(125-150 \mathrm{cGy}$ per dose) and were then evaluated for resection. The delivered preoperative radiation dose was up to 6250 to $7500 \mathrm{cGy}$.

Results: Five patients (14\%) had a partial response to preoperative therapy, and 28 of 37 patients underwent successful resection. There were no intra- or postoperative deaths. Local control was achieved in 19 of 28 resected patients, but in only 1 of 6 patients who remained unresectable despite therapy. With a median follow-up of 5.8 years, $28 \%$ of patients are alive with no evidence of disease, $17 \%$ are alive with disease, and $53 \%$ have died of their disease.

Conclusions: Using the dose and schedule we employed, resection of large soft tissue sarcomas was possible after high-dose radiation delivered in conjunction with IdUrd. Although local control was acceptable, the high rate of distant failure represents a limitation of any local approach to the treatment of large soft tissue sarcomas and suggests the need for integration of this approach with
\end{abstract} an effective systemic therapy.

Key Words: Idoxuridine-Radiation sensitizers--Soft tissue sarcomas-RadiotherapySurgery.

Local control remains an important issue in the management of large soft tissue sarcomas, particularly in sites other than the extremities. ${ }^{1,2}$ Preoperative multimodality treatment protocols have been widely employed in

Received May 1, 1997; accepted October 14, 1997.

From the Departments of Surgery (VKS, JJS, PAS, AEC) and Radiation Oncology (JMR, TSL), University of Michigan Medical Center, Ann Arbor, Michigan.

Dr. Robertson is now with the Department of Radiation Oncology, William Beaumont Hospital, Royal Oak, MI.

Dr. Sussman is now with the Department of Surgery, University of Cincinnati Medical Center, Cincinnati, $\mathrm{OH}$.

Address correspondence to Vernon K. Sondak, MD, Div. of Surgical Oncology, University of Michigan Medical Center, 3306 Comprehensive Cancer/Geriatrics Center, Box 0932, 1500 E. Medical Center Dr. Ann Arbor, MI 48109-0932, USA.

Presented at the 50th Annual Cancer Symposium of The Society of Surgical Oncology, Chicago, Illinois, March 20-23, 1997. the management of large, high-grade extremity sarcomas to avoid the need for amputation. ${ }^{3,4}$ Preoperative treatment of nonextremity sarcomas has been more problematic. The high doses of radiation that appear to be required to improve local control in an extremity (usually in excess of $6000 \mathrm{cGy}$ ) are above the tolerance of normal tissues in nonextremity sites, particularly the intestine. Moreover, evaluations of systemic chemotherapy in patients with nonextremity sarcomas have been quite disappointing. ${ }^{5,6}$ Complete surgical removal remains the mainstay of treatment for large soft tissue sarcomas.

We therefore sought to develop a protocol that would maximize the resectability of large extremity and nonextremity soft tissue sarcomas by increasing the effectiveness of preoperative radiation therapy. Our approach was based on the use of high doses of radiation in conjunction with the radiation sensitizer idoxuridine (IdUrd $\left.{ }^{4}\right)$. IdUrd 
is a thymidine analogue that is incorporated into the DNA of proliferating cells in place of thymidine, increasing the susceptibility of those cells to killing by ionizing radiation. ${ }^{7,8}$ Sensitization by IdUrd is directly proportional to the percentage incorporated into DNA, which is, in turn, proportional to the total delivered dose of drug. The clinical application of halogenated pyrimidine radiosensitizers, however, has been limited by the fact that all proliferating cells incorporate the agents. In large soft tissue sarcomas, the tumor might be adjacent to normal tissues (such as intestine and bone marrow) that would be expected to incorporate thymidine analogs efficiently. Indeed, a disappointingly high level of normal tissue radiosensitization was observed in patients treated with concurrent 5-bromo-2'-deoxyuridine (BrdUrd) and radiation. ${ }^{9}$ Furthermore, these thymidine analogs have intrinsic toxicity following long-term continuous infusion, which limits the total dose of sensitizer that can be delivered. ${ }^{10,11}$

Our strategy for achieving selective radiosensitization of tumor versus rapidly dividing normal tissue involved the use of sequential, alternating administration of IdUrd and radiation. Cytokinetic studies have suggested that the intestinal mucosa and bone marrow replicate more rapidly than do most human solid tumors. ${ }^{12,13}$ Inserting a short treatment break between the end of sensitizer infusion and the beginning of radiation should result in a greater fall-off in the level of incorporation in normal tissue than in tumor cells. This would occur if the normal tissues continued to proliferate more rapidly than the tumor, because the fraction of thymidine replaced by IdUrd would be decreased by newly incorporated thymidine. The expected turnover of normal cells (e.g., migration of mature leukocytes from the marrow or shedding of cells from the intestinal mucosa into the gut lumen) would further contribute to selective radiosensitization with this approach. Laboratory studies in animal models have confirmed that improved selectivity can be achieved with this sequential strategy. ${ }^{14}$ Furthermore, it seemed likely that frequent short-term IdUrd infusions ultimately would permit the delivery of higher total doses with less hematologic toxicity than could be achieved with more prolonged exposures. This hypothesis was supported by cell culture studies, which demonstrated that, whereas radiosensitization depends solely on the extent of incorporation of analog into DNA, ${ }^{7,8,15,16}$ cytotoxicity increases with the duration of incorporation. ${ }^{17}$ Lengthening the duration of treatment with halogenated pyrimidines has been shown to increase systemic toxicity in animal studies as well. ${ }^{13}$

Based on the preclinical data cited above, we initiated a prospective trial in patients with locally advanced or unresectable (or both) soft tissue sarcomas (UMCC 8802). Patients received up to five cycles of therapy, with each cycle consisting of 5 days of continuous infusion IdUrd followed 3 days later by external beam radiation. This was a phase I/II clinical trial, incorporating a dose escalation of the IdUrd. The initial cohort of patients received a dose of $1000 \mathrm{mg} / \mathrm{m}^{2}$ body surface area (BSA) per day, which was escalated in subsequent cohorts to $1333 \mathrm{mg} / \mathrm{m}^{2} / \mathrm{d}$ and then to $1600 \mathrm{mg} / \mathrm{m}^{2} / \mathrm{d}$. In this report, we describe the mature clinical results of this trial, focusing on the endpoints of objective response, resectability, local control, and disease-free and overall survival.

\section{METHODS}

\section{Patient Eligibility}

From April 1, 1989, through October 1, 1995, 38 patients with locally advanced or unresectable soft tissue sarcomas were enrolled onto UMCC 8802. To be eligible, patients had to have a pathologically confirmed primary or recurrent soft tissue sarcoma (excluding Ewing's, osteogenic, and visceral sarcomas) in one of the following categories: (a) large $(>5 \mathrm{~cm})$, intermediate- or high-grade soft tissue sarcomas of the head and neck, trunk, or retroperitoneum; (b) large, intermediate- or high-grade soft tissue sarcomas arising in an extremity and considered unresectable without amputation; or (c) any soft tissue sarcoma considered to be unresectable for cure by standard surgical excision. Virtually all the patients in this series were in categories b or c, as outlined below. Patients could not have received prior chemotherapy or radiation, and had to have undergone no more than an incisional biopsy (i.e., measurable tumor must have been present). All patients were 16 years of age or older, and were required to have adequate organ function, defined as follows: granulocyte count $\geqslant 1500 / \mathrm{mm}^{3}$ and platelet count $\geqslant 100,000 / \mathrm{mm}^{3}$; serum bilirubin $\leqslant 2.0$ $\mathrm{mg} / \mathrm{dL}$; serum creatinine $\leqslant 2.0 \mathrm{mg} / \mathrm{dL}$; and blood urea nitrogen $\leqslant 40 \mathrm{mg} / \mathrm{dL}$.

Unresectability was determined by clinical criteria (physical examination and imaging studies) or by exploratory operation, and was defined as the perceived inability to completely resect all gross tumor with a histologically negative margin. In patients who had not undergone previous surgical exploration, the final determination of resectability was made by a multidisciplinary tumor board including experienced oncologic surgeons before protocol therapy was initiated. Although no single set of criteria was used, our basic prerequisites for considering an extremity tumor "unresectable without amputation" were as follows: nonmobile tumor by physical examina- 
tion, plus tumor abutting more than $50 \%$ of the circumference of the bone or any tumor directly involving the joint, bones, interosseous membrane, or essential neurovascular structures. Nonextremity tumors were considered "unresectable for cure" if they were nonmobile by physical examination and directly abutted bone or major neurovascular structures or organs that could not be resected en bloc with the tumor. It was recognized that some sarcomas meeting the above criteria could be resected leaving microscopic or macroscopic tumor at the margins. Such cases were eligible for participation in this trial.

In accord with the policies of the University of Michigan and the Department of Health and Human Services, all patients signed an informed consent form approved for this purpose by the Institutional Review Board of the University of Michigan Hospital, which indicated that they were aware of the investigational nature of the treatment and the potential risks.

\section{IdUrd Infusion}

IdUrd (idoxuridine, 5-iodo-2'-deoxyuridine, NSC 39661 , also called IUdR) was given by continuous intravenous infusion for 5 days. The dose of IdUrd administered was escalated during the course of the study, as experience at each dose level accumulated. The first eight patients received $1000 \mathrm{mg} / \mathrm{m}^{2} / \mathrm{d}$, the next ten received $1333 \mathrm{mg} / \mathrm{m}^{2} / \mathrm{d}$, and subsequent patients received $1600 \mathrm{mg} / \mathrm{m}^{2} / \mathrm{d}$. In cases of severe (grade 4 hematologic or grade 3 or 4 nonhematologic) toxicity, the IdUrd dose was modified as follows: treatment was delayed until resolution and the dose reduced by $25 \%$ for all subsequent infusions. If a second episode of the same severe toxicity occurred, treatment was again delayed until resolution and the IdUrd dose was reduced a further $25 \%$. If a third episode occurred, the patient was taken off study and received no further IdUrd therapy. Toxicity was graded according to the NCI Common Toxicity criteria.

\section{Radiotherapy}

To prepare for radiotherapy, all patients were immobilized in a foam cradle and a computed tomographic (CT) scan was obtained in the treatment position. Radiation portals were individualized and designed using three-dimensional treatment planning. ${ }^{18}$ The fields encompassed the entire tumor plus a 3- to 6-cm margin during the first three cycles, whereupon the field was narrowed to the entire tumor plus a $1-$ to $1.5-\mathrm{cm}$ margin for the remaining two cycles. The total dose to the spinal cord did not exceed $3900 \mathrm{cGy}$. If more than one half of the kidney parenchyma on one side was treated, the other kidney was excluded from the radiation field. Patients with extremity, mediastinal, or head and neck tumors (i.e., those with no bowel in the radiation field) received external beam radiation as twice daily $150-\mathrm{cGy}$ fractions at least 4 hours apart (cumulative radiation dose for patients completing the entire protocol: $7500 \mathrm{cGy}$ ). Patients with retroperitoneal tumors received radiation as twice daily 125 -cGy fractions at least 4 hours apart (cumulative radiation dose for patients completing entire protocol: $6250 \mathrm{cGy}$ ). Treatments were delivered using 6- to $15-\mathrm{MV}$ photons, 9- to $20-\mathrm{MeV}$ electrons, or both. In cases of toxicity, the radiation dose was not modified, and every effort was made to avoid delays between the end of the IdUrd infusion and the beginning of radiation treatment (i.e., within one cycle). Rather, necessary dose delays were between cycles.

\section{Treatment Scheme}

Three cycles of radiosensitizer and radiation ( 6 weeks of therapy) were administered initially, whereupon all patients were assessed for resectability. Insofar as possible, patients were to undergo tumor resection at that point and receive two additional cycles postoperatively. For some patients, resection still was deemed not feasible after three treatment cycles had been completed. These patients received all five cycles of therapy before resection was attempted. In either event, tumor resection was performed approximately 4 weeks after the last radiation treatment. Once the surgical wound had healed satisfactorily, patients who had received three preoperative cycles of therapy were treated with two additional cycles. Patients who had received five cycles of therapy before undergoing surgery did not receive any additional postoperative therapy. Because of the lack of demonstrated survival benefit in randomized studies at the time this study was conducted, ${ }^{19}$ patients did not receive adjuvant chemotherapy.

\section{Assessment of Response}

Standard criteria for the assessment of response to therapy were employed. Clinical complete response was defined as the complete disappearance of all known disease for at least 4 weeks with no evidence of new disease. Partial response was defined as a decrease in the cross-product of the perpendicular diameters of the tumor by at least $50 \%$, lasting at least 4 weeks and with no evidence of new disease. Progressive disease was defined as an increase in the cross-product of the perpendicular diameters of the tumor by at least $25 \%$, or the development of new disease.

\section{Statistical Analysis}

Comparisons of outcomes between groups were done using $\chi^{2}$ analysis. A two-sided $P$ value of $<.05$ was considered significant. 


\section{RESULTS}

\section{Patient Demographics and Tumor Characteristics}

Thirty-eight patients were enrolled onto this phase I/II clinical trial. One patient was deemed ineligible when review of an outside incisional biopsy failed to confirm the diagnosis of malignancy. All other patients were fully eligible and evaluable for toxicity, response, and resectability. One patient was lost to follow-up postoperatively and is censored from analyses of disease-free and overall survival. The median age of eligible patients on this trial was 49 years (mean 46 years, range 17 to 73 years). There were 16 females and 21 males. All patients had measurable tumor, and none had received previous radiation or chemotherapy. Two patients had locally recurrent tumors after prior surgical therapy; one patient presented with potentially resectable lung metastases concurrently with a retroperitoneal primary tumor. The distribution of tumors by site, grade, and histology is given in Table 1. All tumors were larger than $5 \mathrm{~cm}$ in maximal diameter; 32 of 37 tumors were over $10 \mathrm{~cm}$.

\section{Toxicity and Response to Preoperative Treatment}

All 37 eligible patients were evaluable for toxicity and response to preoperative treatment. The dose-limiting toxicity was significant stomatitis encountered at the $1600 \mathrm{mg} / \mathrm{m}^{2} / \mathrm{d}$ dose level but not at lower doses. This was particularly severe in the two patients with head and neck sarcomas, both of whom required enteral feedings via percutaneous, endoscopically placed gastrostomy tubes. Nausea, vomiting, and diarrhea often were doselimiting in patients with retroperitoneal tumors. Three patients failed to complete preoperative therapy because of toxicity. One patient was taken off study because of prolonged grade III neutropenia and successfully completed preoperative therapy with radiation alone without further IdUrd. She underwent complete resection of her tumor but was lost to postoperative follow-up. One patient with a pelvic retroperitoneal sarcoma developed a

TABLE 1. Tumor characteristics

\begin{tabular}{lrlrlc}
\hline \multicolumn{1}{c}{ Tumor site } & & \multicolumn{2}{c}{ Tumor grade } & \multicolumn{2}{c}{ Histologic subtypes } \\
\hline Extremity & 13 & Grade I & 6 & MFH & 12 \\
Retroperitoneum & 20 & Grade II & 7 & Neurofibrosarcoma & $9^{*}$ \\
Head and neck & 2 & Grade III & 24 & Liposarcoma & 5 \\
Mediastinum & 2 & & & Undifferentiated & 4 \\
& & & & Fibrosarcoma & 3 \\
& & & Leiomyosarcoma & 1 \\
& & & Synovial sarcoma & 1 \\
& & & Other & $2^{* *}$ \\
\hline
\end{tabular}

* Seven in patients with neurofibromatosis type 1.

$* *$ One case each of extraosseous chondrosarcoma and adenosarcoma. grade IV bilirubin increase, and one patient with a very large retroperitoneal sarcoma developed a grade IV bilirubin increase as well as grade IV neutropenia and diarrhea. Both patients completed preoperative therapy with radiation alone; only the latter patient was able to undergo resection. All three patients were considered nonresponders to treatment.

During the 6- to 10 -week preoperative treatment period, four patients developed or had progression of known distant metastases (lung metastases in 3 patients, liver metastases in 1 patient). No patient had progression $(>25 \%$ increase) in the size of the primary tumor during treatment. There were no clinical complete responses, but five patients had partial responses, for a $14 \%$ overall objective response rate. Responses were seen in three of 13 patients with extremity tumors, one of 20 patients with retroperitoneal tumors, and one of four patients with nonextremity, nonretroperitoneal tumors. Most responders (four of five) had grade III tumors, and none had grade I tumors. Although relatively few patients had regression sufficient to qualify as a partial response, most patients had sufficient shrinkage of tumors to permit ultimate resection, as discussed later. Histologic evidence of significant treatment effect, with posttreatment necrosis in $20 \%$ to $100 \%$ of tumor cells, was seen in most patients. Complete tumor necrosis was found in one case, and extensive ( $>98 \%$ ) necrosis was found in another when the tumors demonstrating a partial response were resected. The remaining objective responders had lesser degrees of posttreatment necrosis.

\section{Resectability After Preoperative Treatment}

Only one of four patients who developed distant metastases underwent resection of the treated primary tumor for local control. Of the remaining 33 patients, 27 underwent resection, for a total of 28 of $37(76 \%)$ of all eligible patients. Histologically negative margins were achieved in 13 of the 28 resections (46\%), and gross residual disease was left in only two patients (one of whom had $100 \%$ tumor necrosis and remains alive and continuously disease-free almost 8 years postoperatively), strongly suggesting that in at least some cases preoperative treatment allowed curative resections that would not have been possible otherwise. Six patients remained unresectable despite preoperative treatment, and all but one ultimately progressed in the treated field. Among the 13 patients with extremity sarcomas considered to require amputation in the absence of preoperative treatment, 10 patients underwent successful resection (seven with histologically negative margins), two patients developed distant metastases and never had surgical treatment of their primary tumors, and one patient 
failed to respond sufficiently to permit resection with limb preservation. This patient refused amputation and was treated with further radiation and IdUrd alone; he subsequently died of distant disease with control of his primary tumor.

In no case did the preoperative treatment preclude or significantly hamper the definitive resection. There were no intraoperative or postoperative deaths.

\section{Late Toxicity}

Thirty-six patients were evaluable for late toxicity. One patient with a low-grade retrohepatic sarcoma developed acute cholangitis 18 months after completion of therapy and died with no evidence of residual tumor at necropsy. This was felt to be a late death from toxicity, as the bile duct was within the treated field. Adhesive bowel obstructions necessitating reoperation were seen in two patients, one at 2 months and the other at 3 years after resection of large retroperitoneal sarcomas. No cases of radiation enteritis have been encountered, with a maximum follow-up of 7.5 years from the completion of therapy for the longest surviving patient. In addition to the two reoperations for bowel obstruction, four other patients had late toxicity requiring surgery: one patient's extremity wound dehisced, requiring myocutaneous flap coverage; one patient with a paraspinous sarcoma developed a cerebrospinal fluid leak requiring omental flap closure; one patient with a pelvic sarcoma developed a persistent ureteral stricture, ultimately requiring nephrectomy; and one patient with a groin sarcoma developed a pathologic fracture of the proximal femur requiring open fixation.

\section{Outcome}

With a median follow-up of 5.8 years, and a minimum follow-up of 2.5 years for surviving patients, there have been nine local recurrences among 36 evaluable patients $(25 \%)$. Four of these recurrences developed simultaneous with or subsequent to distant metastasis. Local recurrence was somewhat more common in nonextremity sites, with two local recurrence among 10 resected extremity tumors as opposed to five of 14 resected retroperitoneal tumors and two of three resected head and neck or mediastinal sarcomas. Fourteen of 36 patients are alive $(39 \%)$, but only ten $(28 \%)$ are alive and free of disease. This includes one patient who developed a solitary lung metastasis, which was successfully resected, who remains disease-free 3 years postthoracotomy. Distant metastases developed in $50 \%$ of patients, distributed equally among the different primary sites. Of note, distant disease developed in two of the five patients with large, low-grade sarcomas (all retroperitoneal). Although the total number of patients is too small to adequately assess the influence of histologic subtype on outcome, there was no obvious trend for a better or worse outcome for patients by histologic subtype. For example, two of nine patients with neurofibrosarcomas (both of which arose in conjunction with neurofibromatosis type 1) are alive and continuously disease-free more than 5 years after completion of therapy; this percentage does not differ significantly from the group as a whole (22\% versus $28 \%$ ).

Among the five patients who achieved a partial response, all are alive and four have no evidence of disease, compared to nine alive among the remaining 31 evaluable patients. This difference in overall survival between responders and nonresponders is statistically significant $\left(P_{2}<.02, \chi^{2}\right.$ test $)$. Hence, there appears to be a correlation between response to preoperative treatment and outcome.

\section{DISCUSSION}

Our main goal in conducting this protocol was to intensify local therapy for patients with large soft tissue sarcomas, with the hope that this would improve resectability and local control and perhaps translate into longterm disease-free survival. We were able to successfully resect large extremity and nonextremity sarcomas after significant doses of externally delivered radiation without excessive morbidity or mortality. Ten of 13 extremity sarcoma patients underwent radical resections after 4500 cGy of radiation, delivered in conjunction with the radiosensitizer idoxuridine, with two major local complications and no amputations or deaths. Fourteen of 20 patients with retroperitoneal sarcomas underwent resections after either 3750 or $6250 \mathrm{cGy}$ of radiation plus sensitizer, again with acceptable morbidity and no mortality. One patient with a mediastinal tumor underwent uneventful resection of tumor adjacent to the aorta and esophagus after $7500 \mathrm{cGy}$ of radiation plus sensitizer. Although the scheduling of the radiation (every-otherweek treatment with twice-daily fractions) probably helped to minimize the toxicity of treatment, the administration of a sensitizer and the high cumulative dose of radiation delivered meant that tumors and adjacent normal tissues received very close to maximal amounts of local therapy. Significantly, histologically negative margins were achieved in $46 \%$ of the patients undergoing resection (70\% of the patients with extremity tumors), despite the fact that virtually all patients were felt to have been unresectable to negative margins prior to radiation as a condition of entry onto the study.

This study was not randomized, and was not intended 
to answer questions such as the relative value of preversus postoperative radiation or the benefit of delivering a radiation sensitizer. Idoxuridine recently has been reported to improve survival when used as a radiosensitizer for the treatment of anaplastic astrocytomas. ${ }^{20}$ One large trial of idoxuridine given by continuous infusion concurrently with radiation in patients with unresectable sarcomas has been performed, and revealed local control rates that the authors suggested represented a potential improvement over radiation alone. ${ }^{10}$ There are few, if any, series in the literature evaluating sarcoma patients with characteristics similar to the patients in our study, limiting our ability to compare our results to those of others using different treatment protocols. Nonetheless, a survey of the available literature suggests that our local control and resectability rates are at least comparable to those reported from other centers. For example, Eilber et al. ${ }^{21}$ recently reported on 99 selected patients with highgrade extremity sarcomas treated with preoperative chemotherapy and radiation followed by surgery. They reported a limb-sparing resection rate of $95 \%$, a local recurrence rate of $20 \%$, and a 5-year disease-free survival of $70 \%$, after a median follow-up of 6 years. The average size of the tumors in their series was smaller than that in our study, so our results seem to compare favorably. Casper et al. ${ }^{22}$ reported on 23 patients with high-grade extremity sarcomas treated with preoperative chemotherapy and postoperative radiation with no objective responses seen, a limb-sparing resection rate of $96 \%$, an isolated local recurrence rate of $9 \%$, and a disease-free survival of $41 \%$ after a median follow-up of 39 months.

In contrast to extremity sarcomas, where preoperative, multimodality therapy is widely used, no consensus exists as to whether and how to treat nonextremity sarcomas preoperatively. Most published series have employed resection followed by radiation, chemotherapy, or both, with varying results. Among the best results with such an approach are those reported by Karakousis et al. ${ }^{23}$ with a resectability rate of $95 \%$, a local recurrence rate of $31 \%$, and a 5-year survival of $63 \%$ in 87 consecutively treated patients. Results of other series have not been quite as favorable. Sindelar et al., ${ }^{24}$ in a series of resectable retroperitoneal sarcoma patients, conducted a prospective randomized trial of intraoperative plus postoperative external beam radiation versus postoperative radiation alone. Sixty percent of patients developed local recurrences, with no overall benefit seen for intraoperative radiotherapy and a high incidence of complications of intraoperative treatment. Interestingly, in this series $34 \%$ of patients developed "disabling" radiation enteritis, a complication we did not observe even though we administered a higher total dose of external beam radiation.

Since our protocol was completed, a meta-analysis of the effect of adjuvant chemotherapy on outcome in patients with soft tissue sarcomas has been conducted. ${ }^{25}$ This analysis suggested a small benefit for adjuvant chemotherapy in decreasing local recurrence and improving disease-free and, possibly, overall survival. It remains unclear if the benefits of adjuvant chemotherapy are seen equally in sarcomas arising in all areas, or whether a disproportionate benefit accrues to patients with extremity tumors. Nonetheless, the results of this metaanalysis-particularly when combined with the high rate of distant failure encountered on our protocol-make the incorporation of systemic chemotherapy into multimodality treatment protocols for nonextremity sarcomas an attractive prospect. Since completing our protocol, we have treated a small number of patients with large retroperitoneal sarcomas with preoperative chemotherapy without radiation. With relatively short follow-up, the local recurrence rate appears to be higher than what we saw with our preoperative radiation and radiosensitizer protocol. Consequently, we believe that the integration of both chemotherapy and high-dose radiation will be required in the next generation of multimodality therapy protocols for nonextremity sarcoma patients. It is particularly interesting in this regard to note that IdUrd has been reported to be a potent chemosensitizer in vitro as well as a radiosensitizer, ${ }^{26,27}$ and phase I clinical trials of IdUrd plus chemotherapy already have been conducted. $^{28}$

One additional caveat regarding preoperative chemotherapy must be mentioned. It has been reported that patients who have a good response to preoperative chemotherapy fare better than patients who do not, implying an effect of the chemotherapy on microscopic distant disease. ${ }^{29}$ The treatment regimen we tested in our protocol did not involve systemic antitumor chemotherapy, and the systemic use of a halogenated pyrimidine radiosensitizer would not be expected to provide any direct cytotoxic effects on sarcoma cells. Nonetheless, we saw a statistically significant trend for patients whose tumors responded to therapy to have improved disease-free and overall survival compared to nonresponders. This raises the possibility that responding tumors may be an inherently more favorable group, regardless of the treatment to which they are responding, and calls into question the validity of analysis of responders versus nonresponders as a means for assessing overall efficacy of a given treatment.

In conclusion, we found that a short-term continuous infusion of IUdR and high-dose radiation is tolerable, 
and that this therapy can lead to sufficient regression of locally advanced and even unresectable sarcomas to permit successful resection with acceptable local control rates. The high incidence of distant failure represents a major limitation of this or any local regional approach to very large sarcomas. Nonetheless, we believe our results warrant consideration for the integration of high-dose radiation and radiation sensitizers into modern multimodality therapy as the next logical step. The apparent correlation of response and outcome suggests that caution should be used in concluding there is a systemic effect of any preoperative therapy; we believe that there was none in our trial. Finally, although randomized trials ultimately will be required to determine if radiosensitizers have any role in the treatment of locally advanced sarcomas, we believe the next generation of clinical trials should focus on the integration of these aggressive local approaches with modern systemic treatment.

Acknowledgment: This work was supported by NTH grants CA 53440, CA 44173, CA 42761, and MO1 RR 00042, and Cancer Center Core Grant CA 46592. Dr. Sondak and Dr. Lawrence are recipients of American Cancer Society Career Development Awards. Dr. Robertson is a recipient of an American Society of Clinical Oncology Career Development Award.

\section{References}

1. Emrich LJ, Ruka W, Driscoll DL, Karakousis CP. The effect of local recurrence on survival time in adult high-grade soft tissue sarcomas. $J$ Clin Epidemiol 1989;42:105-10.

2. Rööser B, Gustafson P, Rydholm A. Is there no influence of local control on the rate of metastases in high-grade soft tissue sarcoma? Cancer 1990:65:1727-9.

3. Suit HD, Mankin HJ, Wood WC, et al. Treatment of the patient with stage M0 soft tissue sarcoma. J Clin Oncol 1988;6:854-62.

4. Eilbert F, Eckardt J, Rosen G, Forscher C, Selch M, Fu YS. Preoperative therapy for soft tissue sarcoma. Hematol Oncol Clin North Am 1995;9:817-23.

5. Glenn J, Sindelar WF, Kinsella T, et al. Results of multimodality therapy of resectable soft-tissue sarcomas of the retroperitoneum. Surgery 1985;97:316-25.

6. Glenn J, Kinsella T, Glatstein E, et al. A randomized, prospective trial of adjuvant chemotherapy in adults with soft tissue sarcomas of the head and neck, breast, and trunk. Cancer 1985;55:1206-14.

7. Kinsella TJ, Dobson PP, Mitchell JB, Fornace AJ. Enhancement of $\mathrm{X}$-ray induced DNA damage by pre-treatment with halogenated pyrimidine analogs. Int J Radiat Oncol Biol Phys 1987;13:733-9.

8. McGinn CJ, Kinsella TJ. The clinical rationale for S-phase radiosensitization in human tumors. Curr Probl Cancer 1993;17:275321.

9. Kaplan HS. Radiobiology's contribution to radiotherapy: promise or mirage? Radiat Res 1970;43:460-76.

10. Goffman T, Tochner Z, Glatstein E. Primary treatment of large and massive adult sarcomas with iododeoxyuridine and aggressive hyperfractionated irradiation. Cancer 1991;67:572-76.

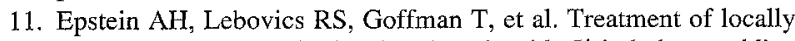
advanced cancer of the head and neck with $5^{\prime}$-iododeoxyuridine and hyperfractionated radiation therapy: measurement of cell labeling and thymidine replacement. $J$ Natl Cancer Inst 1994;86: 1775-80.

12. Ponz de Leon M, Roncucci L, Di Donato P, et al. Pattern of epithelial cell proliferation in colorectal mucosa of normal subjects and of patients with adenomatous polyps or cancer of the large bowel. Cancer Res 1988;48:4121-6.

13. Lawrence TS, Davis MA, Maybaum J, et al. The potential superiority of bromodeoxyuridine to iododeoxyuridine as a radiation sensitizer in the treatment of colorectal cancer. Cancer Res 1992; $52: 3698-704$.

14. Lawrence TS, Davis MA, Stetson PL, Maybaum J, Ensminger WD. Kinetics of bromodeoxyuridine elimination from human colon cancer cells in vitro and in vivo. Cancer Res 1994;54:2964-8.

15. Iliakis G, Kurtzman S, Pantelias G, Okayasu R. Mechanism of radiosensitization by halogenated pyrimidines: effect of $\mathrm{BrdU}$ on radiation induction of DNA and chromosome damage and its correlation with cell killing. Radiat Res 1989;119:286-304.

16. Lawrence TS, Davis MA, Maybaum J, Stetson PL, Ensminger WD. The effect of single versus double stranded substitution on halogenated pyrimidine-induced radiosensitization and DNA strand breakage in human tumor cells. Radiat Res 1991;123:192-8.

17. Lawrence TS, Davis MA, Maybaum J, Stetson PL, Ensminger WD. The dependence of halogenated pyrimidine incorporation and radiosensitization on the duration of drug exposure. Int $J$ Radiat Oncol Biol Phys 1990;18:1393-8.

18. Robertson JM, Sondak VK, Weiss SA, Sussman JJ, Chang AE, Lawrence TS. Preoperative radiation therapy and idoxuridine for large retroperitoneal sarcomas. Int J Radiat Oncol Biol Phys 1995; 31:87-92.

19. Elias AD, Antmann KH. Adjuvant chemotherapy for soft-tissue sarcomas: a critical appraisal. Semin Surg Oncol 1988;4:59-65.

20. Urtasun RC, Kinsella TJ, Farnan N, Del Rowe JD, Lester SG, Fulton DS. Survival improvement in anaplastic astrocytoma, combining external radiation with halogenated pyrimidines: final report of RTOG 86-12, Phase I-II study. Int J Radiat Oncol Biol Phys 1996;36:1163-7.

21. Yang RS, Lane JM, Eilber FR, et al. High grade soft tissue sarcoma of the flexor fossae. Size rather than compartmental status determine prognosis. Cancer 1995;76:1398-405.

22. Casper ES, Gaynor JJ, Harrison LB, Panicek DM, Hajdu SI, Brennan MF. Preoperative and postoperative adjuvant combination chemotherapy for adults with high grade soft tissue sarcoma. Cancer 1994; 73:1644-51.

23. Karakousis CP, Velez AF, Gerstenbluth R, Driscoll DL. Resectability and survival in retroperitoneal sarcomas. Ann Surg Oncol 1996;3:150-8.

24. Sindelar WF, Kinsella TJ, Chen PW, Delaney TF, Tepper JE, Rosenberg SA, Glatstein E. Intraoperative radiotherapy in retroperitoneal sarcomas: final results of a prospective, randomized clinical trial. Arch Surg 1993;128:402-10.

25. Tierney JF, Mosseri V, Stewart LA, Souhami RL, Parmar MK. Adjuvant chemotherapy for soft-tissue sarcoma: review and metaanalysis of the published results of randomised clinical trials. $\mathrm{BrJ}$ Cancer 1995;72:469-75.

26. Russo A, DeGraff W, Kinsella TJ, Gamson J, Glatstein E, Mitchell JB. Potentiation of chemotherapy cytotoxicity following iododeoxyuridine incorporation in Chinese hamster cells. Int J Radiat Oncol Biol Phys 1986;12:1371-4.

27. Chi KH, Kunugi KA, Kinsella TJ. Tododeoxyuridine chemosensitization of cisdiamminedichloroplatinum(II) in human bladder cancer cells. Cancer Res 1994;54:2701-6.

28. Schilsky RL, Janisch L, Berezin F, Mick R, Vogelzang NJ, Ratain MJ. Phase I clinical and pharmacological study of iododeoxyuridine and bleomycin in patients with advanced cancer. Cancer Res 1993;53:1293-6.

29. Pezzi CM, Pollock RE, Evans HL, Lorigan JG, Pezzi TA, Benjamin RS, Romsdahl MM. Preoperative chemotherapy for soft-tissue sarcomas of the extremities. Ann Surg 1990;211:476-81. 